\title{
Ampliación de la
}

\section{Luis Felipe Jiménez \\ fjimenez@eclac.cl}

\section{Jéssica Cuadros}

jcuadros@eclac.cl

Unidad de Estudios

Especiales,

CEPAL \section{cobertura de los \\ sistemas de pensiones en América Latina}

La cobertura del sistema de pensiones en América Latina es insuficiente, pese a las reformas introducidas por varios países. Se destaca la influencia de factores estructurales, como el nivel de desarrollo relativo, la incidencia de segmentos del mercado de trabajo de difícil cobertura, rasgos del diseño de los sistemas y sus incentivos, y factores coyunturales. Dada la decisiva influencia de tales elementos estructurales, la ampliación de la cobertura obligará a combinar adecuadamente regímenes contributivos y no contributivos, para lo cual se formulan lineamientos de política. Con el fin de prevenir la pobreza durante la vejez habrá que recurrir a una combinación de elementos de seguros sociales (basados en el ahorro y el reparto) y de seguridad social, con componentes de financiamiento solidario y tributario. 


\section{I}

\section{Introducción}

El objetivo básico de todo sistema de pensiones es el de proveer ingreso a las personas durante la etapa de su retiro de la vida laboral, generalmente luego de alcanzar cierta condición de elegibilidad (edad, incapacidad laboral, entre otras) que cada sociedad considera apropiada. La forma en que se organice el sistema para financiar esta provisión de ingreso y la definición de las contribuciones y los beneficios son aspectos importantes, pero la eficacia del sistema de pensiones para cumplir su objetivo depende de la inclusión, durante las etapas laboralmente activas, de la población que más tarde será elegible para acceder a sus beneficios. En tal sentido, la cobertura de un régimen es uno de sus aspectos claves.

Evidentemente, la cobertura es sólo uno de los parámetros que permite apreciar la calidad de un sistema de pensiones. Otros factores, como su sostenibilidad financiera, la calidad de sus beneficios y su mayor o menor grado de progresividad, así como la eficiencia de su operación, han sido objeto de análisis por parte de la CEPAL en varios estudios anteriores. ${ }^{1}$

En una primera aproximación, el examen de la eficacia de los sistemas de pensiones de la región a partir de la cobertura de la población objetivo, adoptando para estos efectos los sesenta años como un límite promedio de edad para el retiro, arroja resultados que son, en general, decepcionantes. ${ }^{2}$ En sólo cuatro de los países incluidos en el cuadro 1, como éste muestra en su segunda columna, aparentemente se logra una buena cobertura de la población a los que esos sistemas están dirigidos, mientras que en los 18 países restantes la cobertura es insuficiente. ${ }^{3}$

$\square$ Los autores agradecen los comentarios y sugerencias de Oscar Altimir.

${ }^{1}$ Véase los estudios publicados en la serie Financiamiento del desarrollo, de la CEPAL, así como Uthoff y Szalachman (1991, 1992 y 1993), Schulthess y Demarco (1993), y Acuña e Iglesias (1991). Véase también Acosta y Ayala (2002), Packard y Shinkai (2000), Rabelo (2001) y Superintendencia de Administradoras de Fondos de Pensiones (2002).

${ }^{2}$ Esta es sólo una cifra referencial. Las edades de retiro en la región van desde los 55 a los 60 años para las mujeres y desde los 60 a los 65 años para los hombres.

${ }^{3}$ Las cifras del cuadro 1 deben interpretarse con cautela. En primer lugar, no todas las pensiones se otorgan sobre la base de la edad de retiro, existiendo muchos otros motivos, lo que explica los porcentajes en exceso del 100\%. En segundo lugar, en ciertos casos que
El cuadro 1 también ilustra el importante desafío y las implicaciones financieras del objetivo de cubrir a toda la población retirada. En el caso hipotético de que toda la población retirada (de más de 60 años) fuera sustentada por la población más joven (20-59 años) mediante un sistema que le otorgara un beneficio equivalente al producto per cápita de esta última, la razón entre ambos grupos, representada en la primera columna del cuadro, daría cierto indicio del límite máximo de la transferencia de ingresos, como porcentaje del PIB, que habría que efectuar hacia la población de más edad. ${ }^{4}$ Como allí se aprecia, las cifras son muy significativas, aun cuando las tasas de reemplazo fueran muy inferiores a las implícitas en ese cálculo. Esto pone de relieve la necesidad de un diseño cuidadoso al momento de emprender ampliaciones de cobertura para que, por una parte, se centren los esfuerzos públicos en quienes por diversas razones no están en condiciones de ahorrar para su vejez y, por otra, se establezcan sistemas de ahorro con incentivos apropiados para quienes sí están en condiciones de ahorrar.

En lo que sigue se abordan algunos factores que explican la situación de baja cobertura de los sistemas

no se incluyen en el cuadro no existe un régimen formal de pensiones, por lo cual la cobertura media efectiva en la región es inferior a lo que el cuadro sugiere.

${ }^{4}$ Esta es una aproximación basada en el siguiente esquema simplificado. Si la totalidad del producto proviniese de la población en edad activa (PEEA), que es aquella entre 20 y 59 años, el PIB se podría expresar como $P I B=q a * P E E A$, donde $q a$ es el producto per cápita de esa población. Por otra parte, si no existe déficit en cuenta corriente, el gasto debe ser igual al producto, que corresponde al gasto realizado por la PEEA (incluyendo la sustentación de los menores de 20 años) más aquel efectuado por la población retirada (PRET), que en este ejercicio es la de 60 años o más. Es decir, PIB = $g a^{*}$ PEEA $+g r^{*}$ PRET, siendo $g a$ y $g r$ el gasto per cápita de la población activa y retirada, respectivamente. Juntando ambas identidades se obtiene $q a *^{*}$ PEEA $=g a^{*} P E E A+g r^{*} P R E T$. En consecuencia, la transferencia de ingreso hacia la población retirada se puede escribir como $(q a-g a)^{*}$ PEEA $=g r^{*}$ PRET . Como porcentaje del PIB ello se expresa como $(q a-g a)^{*} P E E A / q a *{ }_{P A}=g r{ }^{*} \mathrm{PRET} / q a *{ }^{*}$ A. Si el gasto de los retirados fuera cubierto mediante una pensión igual al producto per cápita de la población en edad activa (es decir, si $g r=q a$ ), entonces la transferencia de ingresos hacia la población retirada, como porcentaje del PIB, sería igual a PRET/PEEA, tal como se presenta en el cuadro 1. Desde luego, éste es un límite máximo. En general, las tasas de reemplazo (en nuestro caso la razón gr/qa) son menores a uno. 
América Latina: Cobertura de los sistemas de pensiones, por países

(Proporción de beneficiarios, en porcentajes)

\begin{tabular}{lccc}
\hline & $\begin{array}{c}\text { Población de } 60 \text { años y más/ } \\
\text { Población de } 20 \text { a } 59 \text { años }\end{array}$ & $\begin{array}{c}\text { Pensionados/ } \\
\text { Población de } 60 \text { años y más }\end{array}$ & $\begin{array}{c}\text { Pensionados/ } \\
\text { Población total }\end{array}$ \\
\hline Uruguay & 34.5 & 151.8 & 25.8 \\
Brasil & 14.1 & 139.4 & 10.0 \\
Chile & 17.5 & 108.2 & 10.4 \\
Argentina & 27.0 & 104.6 & 13.8 \\
Trinidad y Tabago & 17.0 & 62.7 & 5.6 \\
Guyana & 14.0 & 55.9 & 3.3 \\
Panamá & 14.6 & 45.2 & 3.4 \\
Costa Rica & 14.5 & 35.9 & 2.5 \\
Perú & 14.3 & 34.0 & 2.3 \\
Bolivia & 16.2 & 32.8 & 2.3 \\
Dominica & 25.0 & 31.9 & 3.5 \\
Paraguay & 12.0 & 28.5 & 1.5 \\
México & 12.9 & 26.1 & 1.6 \\
Ecuador & 13.9 & 26.0 & 1.7 \\
Guatemala & 12.5 & 25.4 & 1.4 \\
Nicaragua & 11.2 & 22.3 & 1.0 \\
Colombia & 16.1 & 19.3 & 1.5 \\
Jamaica & 18.8 & 18.8 & 1.7 \\
El Salvador & 14.3 & 14.3 & 0.9 \\
Venezuela & 13.0 & 10.9 & 0.7 \\
Honduras & 12.0 & 7.9 & 0.4 \\
Barbados & 25.0 & 4.6 & 0.7 \\
\hline
\end{tabular}

Fuente: Elaborado por los autores con datos de CEPAL (2002) y de Palacios y Pallarès-Millares (2000).

de pensiones y se formulan lineamientos de política orientados a ampliarla y, por ese medio, contribuir a reducir la incidencia de la pobreza en la población retirada. Esas propuestas intentan conciliar la sostenibilidad financiera indispensable para que esas políticas rindan frutos, con la prevención del surgimiento de incentivos perversos y riesgos morales que en la expe- riencia regional han debilitado a estos sistemas. Como se verá, el análisis lleva a concluir que para satisfacer las necesidades de la población retirada es preciso combinar elementos de seguros sociales (típicamente de ahorro previo) con aquellos de seguridad social, en los cuales se contempla el financiamiento solidario o mediante impuestos de ciertos beneficios básicos.

\section{II}

\section{Factores que afectan la cobertura}

El examen de los factores que determinan el grado de cobertura de los sistemas de pensiones enfrenta un serio problema de escasez y falta de homogeneidad de los datos, por lo cual el análisis descansa necesariamente en una comparación difícil, basada en datos no uniformes y dispersos e ilustrada empleando ciertos casos específicos.

En cuanto a lo que disponen las leyes de cada país, en 25 países de la región todos los trabajadores dependientes deben contribuir a los sistemas de pensio- nes. Sólo en 13 esa obligación se extiende a los trabajadores independientes, y en 10 la contribución de estos últimos es voluntaria. No obstante, como ya fue señalado por Mesa-Lago, Cruz Saco y Zamalloa (1990), existe una gran distancia entre lo que esas leyes disponen y la cobertura efectiva de los sistemas. Para comprender los factores tras la baja cobertura que los datos evidencian, se examinan aquí elementos del entorno en el cual operan los sistemas de pensiones, algunas características de su diseño que se traducen en 
GRAFICO 1

América Latina y el Caribe: Contribuyentes/fuerza de trabajo, a mediados de la década de 1990

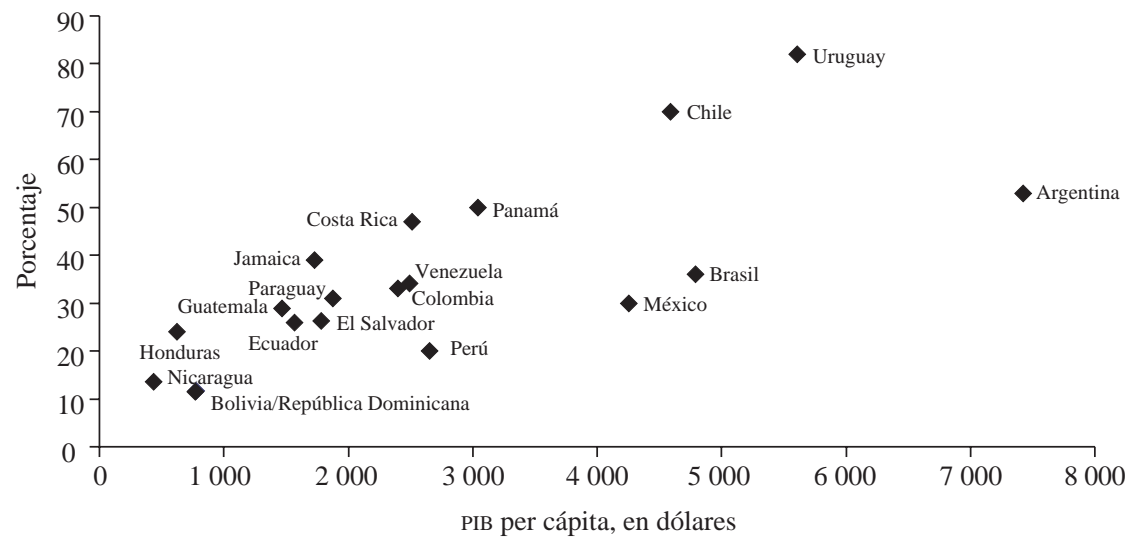

Fuente: elaborado por los autores sobre la base de cifras oficiales y de OIT 2000.

mayores o menores incentivos para participar en ellos, aspectos del mercado de trabajo y características personales que influyen en el resultado.

\section{Factores estructurales}

\section{a) El nivel de desarrollo relativo}

El gráfico 1 ilustra una asociación evidente: los contribuyentes a los sistemas de pensiones aumentan con el nivel del PIB por habitante. Esto se explica básicamente por tres razones. En primer lugar, en general se aprecia cierto avance conjunto en los niveles de ingreso y en la transición demográfica, con lo cual se expande la demanda de mecanismos de provisión de ingresos para la vejez. En segundo lugar, y por el lado de la oferta, a mayor nivel de desarrollo mayor es la disponibilidad de recursos, en especial de carácter público, que pueden ser destinados a organizar un régimen de protección para la población en edad de retiro. En tercer lugar, desde el punto de vista de la estructura económica, la participación del sector formal en la economía y el grado de urbanización se incrementan con el nivel de desarrollo, lo que hace más factible la operación de este tipo de sistemas.

Desde luego, la asociación entre el nivel de producto por habitante y la cobertura de los sistemas de pensiones no implica una causalidad mecánica entre ambas variables, por cuanto el desarrollo de estos sistemas, más que consecuencia espontánea del crecimiento, ha sido resultado de políticas deliberadas orientadas a establecer mecanismos de protección social. b) La importancia de segmentos del mercado laboral de difícil cobertura

La Organización Internacional del Trabajo, en su Panorama Laboral, ${ }^{5}$ presenta evidencias de la baja cobertura de los trabajadores del sector informal urbano. Otro segmento del mercado laboral cuya cobertura es muy difícil es el de los trabajadores independientes y familiares no remunerados de la agricultura. El gráfico 2 presenta información respecto de la importancia en el empleo total de estos dos segmentos y del porcentaje de la población económicamente activa (PEA) que contribuye a la seguridad social, según cifras de la огт. Como allí se aprecia, a medida que crece el porcentaje del empleo que es de difícil cobertura, se reduce el porcentaje de contribuyentes a la seguridad social. ${ }^{6}$

Otro segmento del mercado laboral con baja cobertura, pero que se excluye del concepto anterior, es el de los trabajadores independientes, técnicos y profesionales, que exhiben por lo general un mayor grado de formalización de sus actividades económicas y mayores niveles de productividad. La explicaciones para este fenómeno varían según el caso. Cuando se trata de sistemas basados en el reparto, las razones estriban en que la baja relación entre aportes y beneficios induce a que los aportes sean considerados como impuestos antes que como contribuciones a un esque-

\footnotetext{
5 Véase oIT (2000).

${ }^{6}$ El cuadro 4 presenta estimaciones del empleo de difícil cobertura en relación al empleo total. Este concepto es más amplio que el de sector informal urbano, ya que incorpora a los trabajadores por cuenta propia y familiares no remunerados de la agricultura.
} 


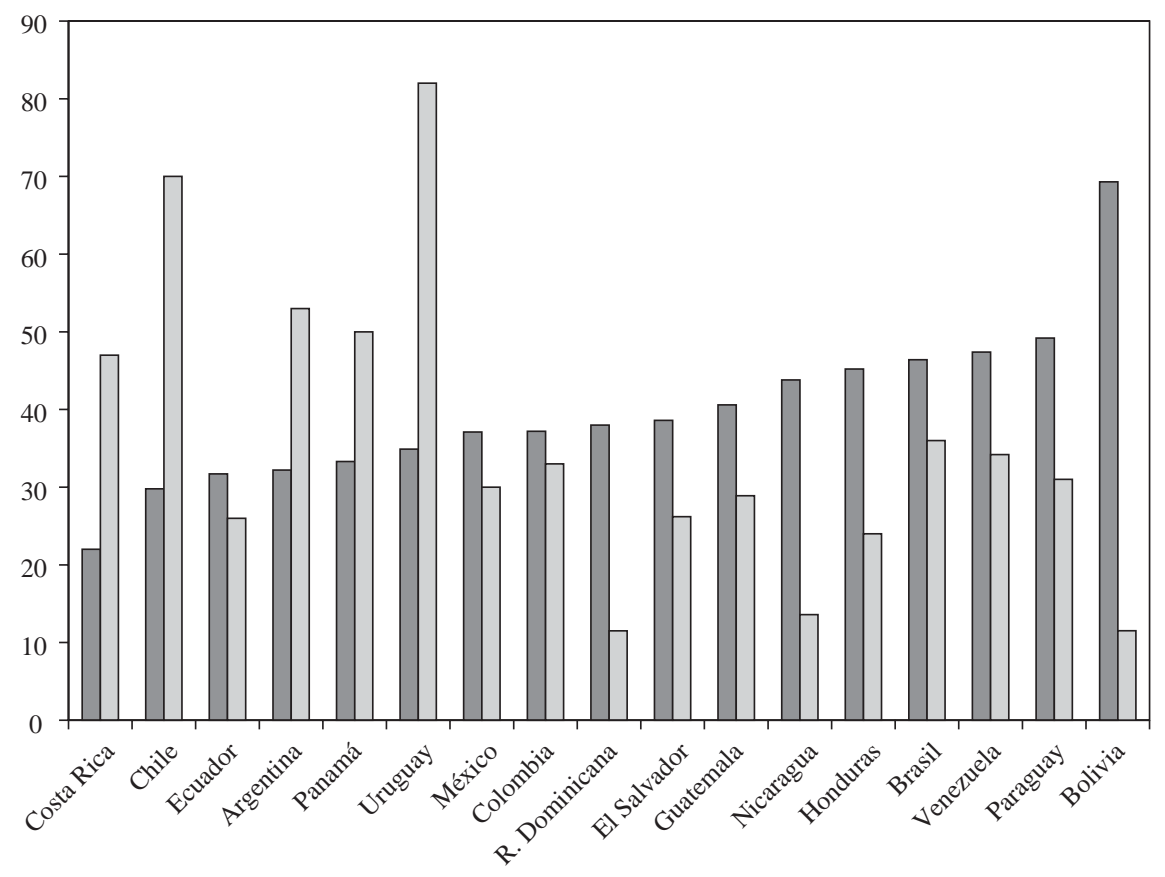

$\square$ Empleo de difícil cobertura $\square$ Contribuyentes/PEA

Fuente: elaborado por los autores.

ma de provisión de ingresos futuros. Como es muy difícil fiscalizar el cumplimiento en este segmento, y/o los aportes son voluntarios, inclusive estos trabajadores más calificados optan por otras formas de asegurar sus ingresos futuros.

En el caso de sistemas basados en el ahorro, para los trabajadores independientes, técnicos y profesionales resultaría más atractivo, en el plazo inmediato, destinar recursos al desarrollo de su propia actividad productiva antes que contribuir a sistemas cuyos retornos son inciertos y cuya liquidez es baja, ya que no ofrecen posibilidades de reasignar los recursos aportados para orientarlos hacia nuevas actividades o para evitar riesgos indeseados.

El carácter irrevocable de los aportes está en la base de este desincentivo, en especial en el caso de sistemas de capitalización, cuyos retornos son inciertos como consecuencia de la variabilidad del entorno macroeconómico y de las fluctuaciones que caracterizan los mercados financieros. Es altamente improbable que una mayoría de estos trabajadores se incline a aportar irrevocablemente a sistemas que están expuestos a sufrir episodios de pérdidas reales. Inclusive, para trabajadores dependientes, cuya afiliación y aportes son obligatorios, la exposición a este riesgo es involuntaria y, probablemente, no deseada. En ambos casos, la exposición forzada a riesgos otorga carácter de impuestos a los aportes previsionales. La inequidad de lo anterior es aún más evidente cuando los operadores privados de los fondos de pensiones no están expuestos a compartir esas pérdidas, aunque su gestión pueda ser deficitaria.

Además, las elevadas comisiones para cubrir costos de administración establecidas por algunos sistemas recientemente introducidos agravan aún más el dilema que enfrentan estos trabajadores. En efecto, el alto nivel de las comisiones desincentiva la afiliación $\mathrm{y}$ aporte de quienes pueden optar entre destinar parte de sus ingresos a un sistema que, por efecto de las comisiones, sólo traduce parcialmente sus aportes totales en un mayor fondo acumulado, o bien confiar en la rentabilidad que ellos mismos podrían obtener 
mediante su propia gestión productiva o alternativas financieras más eficientes.

\section{c) Inserción laboral desventajosa de las mujeres}

Las características del sector informal, junto con la desventajosa inserción laboral de las mujeres, se combinan para dar origen a una situación previsional de precariedad. Para entenderla mejor, resulta útil distinguir tres etapas.

En primer lugar se hallan los factores presistema de pensiones. De ellos, el más importante es la participación considerablemente menor de las mujeres en la fuerza de trabajo, diferencia que es mucho más aguda en los estratos de menores ingresos (gran parte de las mujeres de estos estratos son trabajadoras familiares no remuneradas). Entre las causas que originan esta situación están: mayor peso del cuidado de los niños en los estratos de menores ingresos, niveles educacionales más bajos que reducen el beneficio marginal de incorporarse al mercado de trabajo, y falta de arreglos institucionales al nivel local para hacer frente al cuidado de los menores (Jiménez y Ruedi, 1998).

Las mujeres que durante toda su vida permanecen al cuidado de su casa y familia sin participar en el mercado laboral constituyen un grupo muy expuesto a caer en situación de pobreza, dado que por ser generalmente consideradas como dependientes de sus cónyuges, sólo pueden aspirar a una pensión de viudez.

En segundo lugar están los efectos de carencias en el diseño de los propios sistemas de pensiones, unidos a una inserción laboral desfavorable. Así, cuando las mujeres acceden al mundo laboral lo hacen en mayor proporción en los segmentos informales y de baja remuneración: obtienen empleos intermitentes o temporales, como empleadas domésticas, y empleos de baja calidad, una de cuyas características es el incumplimiento de las leyes laborales. ${ }^{7}$ Como consecuencia, su adscripción a los sistemas formales de pensiones es baja, discontinua y, dados sus menores niveles de ingreso, les proporciona menores beneficios.

Es preciso anotar que este conjunto de circunstancias no tiene relación con que el sistema de pensiones sea público de beneficio definido, o sea privado de capitalización individual. En ambos casos estos fenómenos están presentes, lo que sugiere que en general los sistemas no han sido diseñados para enfrentar la importante desigualdad en las oportunidades de acce-

\footnotetext{
${ }^{7}$ Véase CEPAL, 2000, Anexo estadístico.
}

der a los mecanismos de previsión que opera en contra de la mujeres.

En tercer lugar se encuentran los factores postsistema, o factores de acumulación de derechos, los que difieren según el tipo de sistema de que se trate. En los sistemas de beneficio definido, la mayor desventaja proviene de los menores ingresos que obtienen las mujeres y de un historial de trabajo menos continuo. En los sistemas de capitalización individual y administración privada se agregan otros factores. Por la menor densidad de cotizaciones de las mujeres y sus menores niveles de ingreso, su acumulación de recursos es más baja que en el caso de los hombres. Este hecho se ve agravado por dos circunstancias: la edad de retiro de las mujeres suele ser más baja y su esperanza de vida mayor que la de los hombres. De ese modo, un régimen que vincula cercanamente aportes y beneficios -igualando el valor presente de ambos, como en el caso de una pensión vitalicia ofrecida por un sistema de seguro privado-, necesariamente ofrecerá una menor pensión a las mujeres. Y así sucede aun cuando las mujeres acumulen tantos recursos como los hombres, dada su mayor esperanza de vida y su retiro más temprano.

En suma, la situación de las mujeres es estructuralmente desfavorable en lo que toca a los sistemas de previsión. Para corregirla se necesita que, a través de modificaciones en su diseño, esos sistemas reconozcan las desventajas que enfrentan las mujeres para acceder a las oportunidades que ellos ofrecen, situación que desalienta su afiliación a los sistemas formales.

\section{Rasgos del diseño de los sistemas e incenti- vos resultantes}

\section{a) Las contribuciones como ahorro o impuesto}

En un plano distinto a las consecuencias de las variables examinadas antes y relacionadas con el entorno en que operan los sistemas de pensiones, es también necesario considerar características del diseño de estos sistemas que influyen en su cobertura. De particular interés, más por su importancia en el debate sobre estrategias para la reforma que por su efecto real sobre la cobertura, es el examen de los incentivos para el ahorro previsional que resultan de regímenes de reparto, comparados con aquellos derivados de regímenes de capitalización individual.

En efecto, según una visión ampliamente difundida, la insuficiente cobertura sería inherente a los regímenes de reparto, con baja relación entre aportes y beneficio: los aportes para pensiones habrían sido con- 
siderados más como impuestos que como ahorro para la vejez, lo que habría inducido a la evasión y baja afiliación. En cambio un régimen de capitalización individual, que por definición vincula aportes con beneficios, cambiaría radicalmente la naturaleza de las cotizaciones, transformándolas en ahorro e incentivando así una mayor cobertura.

Esta visión sirvió de punto de apoyo para varios esfuerzos de reforma. Sin abordar el análisis de las relaciones que teóricamente vinculan el ahorro a sus incentivos ni los méritos de adoptar un régimen de capitalización para garantizar la solvencia del sistema, puede afirmarse que la escasa evidencia empírica existente no permite validar la hipótesis levantada en las exégesis de estos regímenes. ${ }^{8}$

En efecto, si se examina la experiencia chilena, caso prototípico en la región, se aprecia que la cobertura total no ha variado sustancialmente en el conjunto de ambos regímenes (cuadro 2). Como allí se aprecia, no obstante la introducción del sistema de Administradoras de Fondos de Pensiones (AFP) y del cierre a nuevos entrantes del Instituto de Normalización Previsional (INP), la cobertura total no excede la que

CUADRO 2

Chile: Evolución de la cobertura del sistema de pensiones (Porcentajes)

\begin{tabular}{|c|c|c|c|c|c|c|}
\hline \multirow[t]{2}{*}{ Año } & \multicolumn{3}{|c|}{ Cotizantes/Ocupados } & \multicolumn{3}{|c|}{ Cotizantes/PEA ${ }^{\mathrm{a}}$} \\
\hline & AFP & INP & Total & AFP & INP & Total \\
\hline 1975 & --- & 71.2 & 71.2 & --- & 61.9 & 61.9 \\
\hline 1976 & --- & 65.7 & 65.7 & --- & 57.3 & 57.3 \\
\hline 1978 & --- & 56.6 & 56.6 & --- & 48.5 & 48.5 \\
\hline 1980 & --- & 53.3 & 53.3 & --- & 47.8 & 47.8 \\
\hline 1982 & 36.0 & 16.6 & 52.6 & 29.0 & 13.4 & 42.3 \\
\hline 1984 & 40.6 & 13.7 & 54.3 & 35.0 & 11.8 & 46.8 \\
\hline 1986 & 45.9 & 11.6 & 57.5 & 41.1 & 10.4 & 51.6 \\
\hline 1988 & 50.6 & 9.8 & 60.4 & 46.6 & 9.0 & 55.6 \\
\hline 1990 & 50.6 & 8.1 & 58.7 & 46.8 & 7.5 & 54.4 \\
\hline 1992 & 55.3 & 6.9 & 62.2 & 51.8 & 6.5 & 58.3 \\
\hline 1994 & 56.2 & 5.5 & 61.7 & 51.8 & 5.0 & 56.9 \\
\hline 1996 & 58.9 & 4.9 & 63.8 & 55.7 & 4.6 & 60.4 \\
\hline 1998 & 58.0 & 4.2 & 62.2 & 53.8 & 3.9 & 57.7 \\
\hline 1999 & 60.4 & 4.2 & 64.6 & 55.0 & 3.9 & 58.9 \\
\hline
\end{tabular}

Fuente: Arenas de Mesa y Hernández (2001).

a PEA: Población económicamente activa.

\footnotetext{
${ }^{8}$ Schmidt-Hebbel (1998) y Uthoff (2001) presentan visiones distintas de los efectos sobre el ahorro que ha tenido la reforma del régimen de pensiones en Chile. A su vez, Fox y Palmer (2001) analizan alternativas de diseño que también se orientan a proteger la solvencia del sistema de pensiones.
}

existía cuando sólo operaba el sistema de reparto. Más bien, la evolución de la cobertura refleja el comportamiento de la economía, con reducciones durante períodos recesivos y ampliaciones en períodos expansivos.

Lo anterior, desde luego, no menoscaba la importancia de emplear criterios de diseño que apunten a vincular aportes y beneficios como mecanismo de protección de la solvencia, al tiempo que se intenta enviar señales correctas para el ahorro; pero no cabe duda de que la experiencia chilena debiera contribuir a moderar las expectativas respecto a la eficacia de este tipo de regímenes para ampliar la cobertura.

\section{b) Incentivos inconsistentes causados por beneficios generosos pero insostenibles}

Aun cuando no se disponga de resultados estadísticos robustos, cabe señalar que el diseño de algunos sistemas resulta en incentivos inadecuados que podrían reducir la afiliación. Así, algunos regímenes de seguridad social otorgan beneficios no sólo al cónyuge e hijos menores de edad, sino que los extienden a otros miembros de la familia en edad activa. El hecho de que los programas de salud y pensiones no sean separables, unido a la extensión de los beneficios a familiares en edad activa, induce a que sólo un miembro de la familia se afilie, por cuanto los beneficios marginales de la incorporación de otro familiar son menores que los costos. Esto es, en el corto plazo existe un incentivo a nivel personal para efectuar la cotización, pero un desincentivo a nivel familiar (Hernández Licona, 2001).

Estas prácticas, que se explican por las diferentes realidades nacionales, afectan la estabilidad financiera del sistema, ya que al reducir el número de contribuyentes e incrementar el de beneficiarios llevan a una ampliación insostenible de la cobertura. En ciertos casos hay además un incentivo implícito a desempeñarse en el sector informal, cuando los beneficios se extienden a familiares en edad productiva que carecen de empleo aparente en el sector formal de la economía pero que laboran en el sector informal.

\section{c) Deficiencias en la "portabilidad" de los fondos de los trabajadores}

Otro elemento que causaría una baja cobertura es que los trabajadores no puedan traspasar sus fondos desde un sistema de pensiones a otro sin perder los beneficios, y que para tener derecho a cobrar una pensión deban tener cierto período de cotización mínimo, pero sin que se contabilicen apropiadamente ni el tiempo de contribución ni los aportes en otros sistemas. 
Bajo esas circunstancias es previsible que los trabajadores preferirán no incorporarse a los sistemas, que se reducirá la movilidad del trabajo y que se incentivará la actividad informal.

Este aspecto ha estado muy presente en las reformas introducidas en la región, las que por diferentes medios, como la creación (y reconstrucción) de registros individuales de la historia laboral y de aportes, han buscado incrementar la portabilidad.

\section{d) Confiabilidad del sistema}

Frecuentemente se argumenta que la percepción adversa respecto de la capacidad para otorgar beneficios de los sistemas de reparto también habría minado la afiliación. Diversas circunstancias —entre ellas el deterioro del valor real de las prestaciones como resultado de procesos inflacionarios y de posteriores ajustes presupuestarios, así como las evidencias de ineficiencia y excesiva demora- se habrían traducido en baja credibilidad del sistema y desincentivos para la participación.

Este argumento contribuyó mucho al énfasis puesto en la privatización total o parcial de los sistemas en algunos países de la región. La hipótesis que lo sustenta es que la gestión privada de los fondos previsionales independizaría al sistema de pensiones de las decisiones políticas, mejoraría su eficiencia y realizaría una buena asignación de los recursos acumulados, fortaleciendo de ese modo la credibilidad del sistema y la confianza en él. Los aspectos de eficiencia y de asignación de fondos, temas muy extensos que exceden el propósito de este artículo, fueron abordados por Rodríguez y Durán (2000) y Mastrángelo (1999). Se desprende de su análisis que las reformas introducidas no han logrado mejorar sustancialmente los aspectos de eficiencia y que éstos no parecen incidir significativamente en la decisión de las personas de afiliarse a los nuevos sistemas. Además, según muestra la experiencia reciente, la independencia del sistema y su protección respecto de presiones políticas no resulta necesariamente de su segregación del aparato estatal, sino que es consecuencia de la solidez institucional y económica general. Situaciones coyunturales adversas pueden afectar la situación de regímenes previsionales supuestamente independientes. ${ }^{9}$

\section{Miopía respecto de las necesidades de ahorro para la vejez}

Una de las hipótesis para explicar el bajo grado de cobertura de los sistemas de pensiones se apoya en la falta de una apreciación clara de la necesidad de ahorrar para la vejez. Esta es una de las razones principales que han llevado a hacer obligatoria la participación en tales sistemas. El cuadro 3 ilustra este comportamiento miope, empleando datos de México. Como allí se ve, la participación en los institutos de seguridad social es baja en las primeras edades laborales y alcanza su máximo en el tramo de edad de 26 a 35 años. Las cifras indican que inicialmente la categoría ocu-

CUADRO 3

México: Categoría ocupacional según la edad

(\% de la PEA)

\begin{tabular}{|c|c|c|c|c|c|c|}
\hline Edad & $\begin{array}{l}\text { Asalariados con } \\
\text { IMSS o ISSSTE }^{\mathrm{a}}\end{array}$ & $\begin{array}{l}\text { Asalariados sin } \\
\text { IMSS o ISSSTE }\end{array}$ & Patrones & Cuenta propia & $\begin{array}{l}\text { Trabajadores } \\
\text { sin pago }\end{array}$ & Total \\
\hline $12-15$ & 2.5 & 36.6 & 0.0 & 3.8 & 57.2 & 100.0 \\
\hline $16-25$ & 28.1 & 42.4 & 1.1 & 8.3 & 20.1 & 100.0 \\
\hline $26-35$ & 37.7 & 29.3 & 4.3 & 21.1 & 7.6 & 100.0 \\
\hline $36-45$ & 32.5 & 25.2 & 6.3 & 29.9 & 6.0 & 100.0 \\
\hline $46-55$ & 25.0 & 22.2 & 8.3 & 37.2 & 7.4 & 100.0 \\
\hline $56-65$ & 15.4 & 16.8 & 8.2 & 53.2 & 6.4 & 100.0 \\
\hline $66-75$ & 5.0 & 11.0 & 8.7 & 67.3 & 8.0 & 100.0 \\
\hline 76 o más & 2.2 & 13.5 & 6.2 & 72.8 & 5.3 & 100.0 \\
\hline
\end{tabular}

Fuente: G. Hernández Licona, 2001.

a IMSS: Instituto Mexicano de Seguridad Social; ISSSTE: Instituto de Seguridad y Servicios Sociales de los Trabajadores del Estado.

\footnotetext{
${ }^{9}$ En Argentina, como parte de los esfuerzos para enfrentar la crisis, el gobierno debió recurrir a los recursos acumulados en los fondos de pensiones privados, forzándolos a una tenencia obligatoria de
}

títulos públicos para satisfacer las necesidades de financiamiento del sector público en 2001. 
pacional más frecuente es la de asalariados con y sin cobertura de la seguridad social. En los tramos de edad más altos se aprecia una reducción de ambos tipos de asalariados, por cuanto los trabajadores independientes incrementan su importancia e inclusive exceden el $50 \%$ de la de la población económicamente activa. Esto se explicaría porque los trabajadores asalariados acumulan experiencia y capital financiero en sus primeros años en el mercado laboral, lo cual les permite más tarde establecer actividades independientes, pasando a convertirse en trabajadores por cuenta propia.

\section{Factores coyunturales: el nivel de empleo}

La cobertura también se ve afectada por factores de corto plazo, si bien esta relación es difícil de documentar dada la escasez de estadísticas apropiadas. Así, la experiencia chilena entre 1981 y 1992, ilustrada en el gráfico 3, muestra que cuando la tasa de desempleo se eleva, se reduce el porcentaje de la población económicamente activa (PEA) que cotiza en los sistemas de pensiones. Una situación similar es informada por Hernández Licona (2001) para el caso de México.

La relación descrita se explica, naturalmente, por la pérdida de vínculo con las instituciones de previsión social que acarrea la situación de desempleo. No hay en la región mecanismos de protección suficientes para contrarrestar esta vinculación. Una alternativa que hoy se discute es la de seguros de desempleo que contemplen la continuidad de los aportes a la seguridad social.
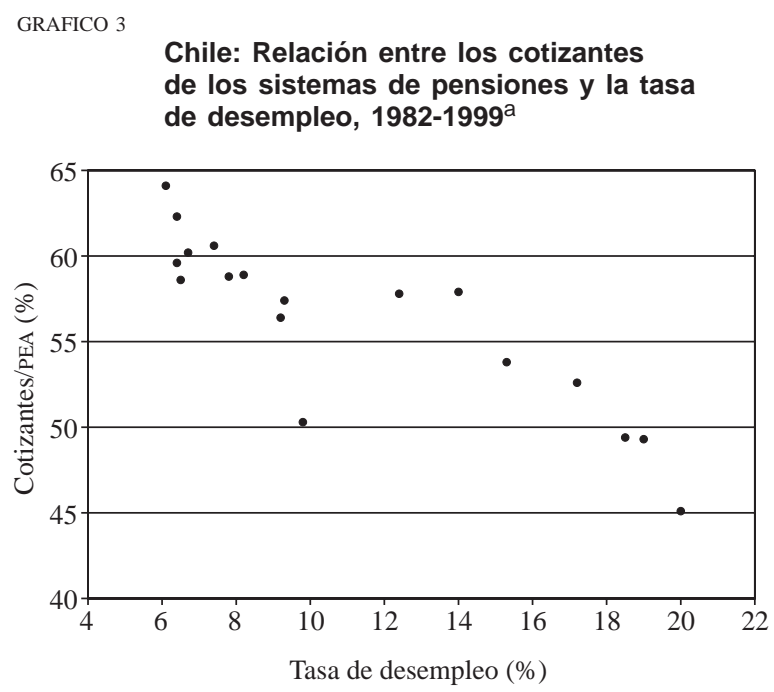

Fuente: Elaborado por los autores sobre la base de cifras del Instituto Nacional de Estadística (INE) y de la Superintendencia de Administradoras de Fondos de Pensiones.

a Incluye los cotizantes del Instituto de Normalización Previsional (INP) y de las Administradoras de Fondos de Pensiones (AFP).

\section{III}

\section{Ampliación de la cobertura de los sistemas}

\section{La magnitud del problema}

Se desprende de lo expuesto en la sección precedente que los progresos en materia de cobertura han sido insuficientes en la región, no obstante las reformas que varios países han introducido en sus sistemas de pensiones. Dada la influencia de factores estructurales en esta situación, las perspectivas de ampliación espontánea de la cobertura no son muy favorables, para regímenes tanto públicos como privados. La cobertura de aquellos grupos que actualmente no se incorporan o no pueden incorporarse a los sistemas previsionales requerirá entonces una acción pública deliberada, basada en el reconocimiento de factores específicos que sistemáticamente los excluyen de los actuales sistemas. Ello requiere, como aquí se argumenta, una adecuada combinación de regímenes contributivos y no contributivos. Aún más, se postula aquí que los regímenes no contributivos deben orientarse principalmente a los sectores pobres sin capacidad de ahorro.

Los grupos generalmente excluidos, a los cuales debiera dirigirse prioritariamente la expansión de la cobertura, son los trabajadores independientes urbanos, tanto del sector formal como del informal, y aquellos del área rural. Además, dentro de estos grupos la situación de la mujer aparece como aún más desfavorecida. En general, los sistemas de pensiones enfrentan serias dificultades para incorporar a estos grupos $\mathrm{y}$, dada la creciente participación del sector informal en el empleo no agrícola en la década de 1990, esas dificultades han tendido a aumentar. Para ilustrar la magnitud del desafío, el cuadro 4 muestra la importancia de los 


\begin{tabular}{|c|c|c|c|}
\hline \multirow{2}{*}{ Países } & \multicolumn{3}{|c|}{$\begin{array}{l}\text { Imérica Latina: Magnitud del empleo } \\
\text { e difícil cobertura por los sistemas } \\
\text { e pensiones, por países, 1998-1999a } \\
\text { Porcentaje del empleo total. Países ordenados } \\
\text { egún PIB per cápita de 1995) }\end{array}$} \\
\hline & $\begin{array}{c}\text { Empleo urbano } \\
\text { de difícil } \\
\text { cobertura }^{b}\end{array}$ & $\begin{array}{c}\text { Empleo agrícola } \\
\text { de difícil } \\
\text { cobertura }^{\mathrm{c}}\end{array}$ & $\begin{array}{c}\text { Empleo total } \\
\text { de difícil } \\
\text { cobertura }\end{array}$ \\
\hline Argentina & 32.2 & n.d. & 32.2 \\
\hline Uruguay & 34.9 & n.d. & 34.9 \\
\hline Chile & 25.9 & 3.9 & 29.8 \\
\hline Brasil & 33.3 & 13.2 & 46.5 \\
\hline México & 30.1 & 6.9 & 37.0 \\
\hline Venezuela & 43.5 & 4.0 & 47.4 \\
\hline Panamá & 20.5 & 12.7 & 33.3 \\
\hline Costa Rica & 17.3 & 4.7 & 22.0 \\
\hline Colombia & 29.3 & 7.9 & 37.2 \\
\hline Paraguay & 27.5 & 21.7 & 49.2 \\
\hline El Salvador & 27.8 & 10.8 & 38.6 \\
\hline Ecuador & 31.7 & n.d. & 31.7 \\
\hline R. Dominicana & 26.8 & 11.2 & 38.0 \\
\hline Guatemala & 20.5 & 20.1 & 40.6 \\
\hline Bolivia & 36.6 & 32.7 & 69.3 \\
\hline Honduras & 24.5 & 20.7 & 45.2 \\
\hline Nicaragua & 26.0 & 17.8 & 43.8 \\
\hline
\end{tabular}

Fuente: CEPAL (2001).

a Países ordenados según su PIB per cápita de 1995.

b Trabajadores no profesionales ni técnicos de establecimientos privados de hasta cinco personas y por cuenta propia, trabajadores familiares no remunerados y empleados domésticos.

c Trabajadores por cuenta propia y familiares no remunerados de la agricultura.

segmentos del mercado laboral de difícil cobertura como proporción de la población ocupada. Como allí se aprecia, en los países de mayor desarrollo relativo de la región aproximadamente el $30 \%$ de los empleos es de difícil incorporación a los sistemas previsionales. En los países más pobres esta proporción suele exceder el $45 \%$ del empleo total, llegando a un máximo de $69 \%$ en Bolivia.

\section{Algunos criterios generales para políticas de expansión de la cobertura de sistemas de pensiones públicos contributivos y no contributivos}

Varias son las propuestas que pueden formularse para ampliar la cobertura, por lo que parece necesario formular ciertos criterios generales antes de discutir lineamientos específicos. En su gran mayoría esos criterios se refieren a regímenes públicos, dado que por las magnitudes y características de la población no cubierta serán ellos los llamados a evitar la pobreza de los segmentos desprotegidos. Sin embargo, el diseño del sistema debe ser cuidadoso para evitar el desincentivo a participar en sistemas basados en el ahorro.

Dado que la mayoría de los factores causantes de una baja cobertura son de largo plazo, para tener efectos duraderos toda política de expansión que se proponga debe ser sostenible. Por lo tanto, el sistema debe estar diseñado para soportar cargas fiscales acordes con la capacidad de largo plazo para generar recursos públicos y destinarlos a este fin. Los programas de ampliación de la cobertura insuficientemente financiados o basados en bonanzas transitorias de los recursos públicos no son sostenibles y su impacto no durará. Así como en el ejercicio de preparación del presupuesto público en varios países se procede a estimar los componentes estructurales y cíclicos de gastos e ingresos, lo mismo debe hacerse en el caso de los sistemas de pensiones para establecer posibilidades duraderas de mayor cobertura.

Por las mismas razones, no es recomendable ampliar sistemas actuarialmente deficitarios, ya que en esos casos el aumento del número de afiliados suele aportar aún más al déficit, haciendo que los beneficios y la mayor cobertura sean insostenibles en el tiempo. En consecuencia, el equilibrio actuarial debiera ser considerado como un requisito previo para la ampliación de la cobertura.

Si bien la universalidad de la cobertura es el objetivo final, en el camino para alcanzarla se debe siempre velar por que quienes puedan cubrir sus necesidades de consumo en la vejez sobre la base de su propia capacidad de ahorro se vean alentados a hacerlo, permitiendo así que los escasos recursos públicos se orienten a los grupos de población que no tienen capacidad de ahorro. Por ello, debe incentivarse la contribución al ahorro para la vejez, pero el nivel de las garantías públicas -en la forma de pensiones mínimas garantizadas para los adscritos a los regímenes públicos contributivos- debe ser cuidadosamente establecido, para evitar el riesgo moral de inducir a cotizar sólo hasta cierto número de años, y que se evada luego la cotización mediante la informalización del empleo. Es preciso mantener permanentemente el incentivo a la contribución, por ejemplo, ofreciendo garantías inicialmente muy bajas, para luego reconocer cada año adicional (o número determinado de años, como bienios o trienios) con incrementos marginales de la garantía que, al menos desde el punto de vista teórico, sean levemente superiores a la utilidad marginal del ocio.

En el caso de beneficios básicos otorgados por regímenes no contributivos, el nivel de estos beneficios 
debe evitar el desincentivo a trabajar una vez que se ha logrado cierta edad o condición de elegibilidad. El necesario límite superior a este beneficio debe establecerse equilibrando dos criterios, según la realidad de cada país. Si el objetivo básico es evitar la pobreza en la vejez, el beneficio no puede exceder la línea de pobreza estimada para cada país. Si el objetivo es mantener el incentivo al trabajo, el límite máximo debe ser inferior al salario mínimo, siempre que éste sea adecuadamente calculado en una magnitud cercana al salario de reserva del trabajo no calificado, dado que este estrato laboral sería el que obtendría tal beneficio. ${ }^{10}$

Otra razón para evitar beneficios generosos en un régimen no contributivo es el desincentivo que ellos significan para la afiliación y cotización en los regímenes contributivos, haciendo recaer en el Estado la responsabilidad de velar por el bienestar de personas que podrían haber contribuido a ahorrar para su vejez. Por lo tanto, la expansión de estos regímenes ha de ser cuidadosamente graduada, mediante la focalización de los beneficios no contributivos en aquellos segmentos que objetivamente no puedan efectuar ese ahorro. Esto puede hacerse, por ejemplo, a través de una prueba de medios (de ingresos y activos), mecanismos de autoselección o tratamientos tributarios que compensen efectos distributivos no deseados de un beneficio universal.

Debe tenerse presente además que los sistemas de pensiones se orientan a la provisión de ingresos para la vejez con el fin de garantizar cierto nivel de consumo. Sin embargo, la satisfacción de esas necesidades de consumo no siempre se logra del modo más eficiente a través de la provisión de ingresos monetarios. Más aún, la necesidad de que los programas sean sustentables en el tiempo para posibilitar efectos duraderos, lleva a que ciertas necesidades de consumo de la población no cubierta por los regímenes contributivos sean satisfechas con más eficiencia mediante la provisión directa de algunos bienes y servicios, como es el caso de la salud y el acceso a medicinas. Los regímenes no contributivos de pensiones deben, en consecuencia, ser considerados como uno de los eslabones que componen la red de protección social más que como una alternativa a los regímenes de carácter contributivo.

Por último, pero no menos importante, los elementos de subsidio que haya que introducir deben te-

\footnotetext{
${ }^{10}$ En varios países de la región el salario mínimo se determina con otros criterios, por lo que su utilización para establecer pensiones mínimas puede originar fuertes distorsiones.
}

ner en lo posible un carácter progresivo o bien ser neutros en términos distributivos.

\section{Algunos lineamientos de política}

\section{a) Rediseñar los sistemas previsionales}

Una importante crítica es la que señala que en la mayoría de los países de la región los regímenes de previsión social siguieron las líneas de sistemas introducidos anteriormente en países desarrollados, que exhiben un mayor grado de urbanización y más actividad económica formal. Una de las alternativas que se postulan para lograr una cobertura universal en nuestros países es la creación (o ampliación, según el caso) de un régimen no contributivo que otorgue un beneficio básico a todos quienes cumplan con determinados requisitos, como edad y residencia en el país. ${ }^{11}$ Este régimen universal no sustituiría a los regímenes contributivos vigentes. Las ventajas que ofrecería serían la cobertura expedita de toda la población y bajos costos de operación. Los posibles efectos adversos en términos de desincentivo al ahorro y al trabajo serían paliados mediante una fijación del beneficio en un nivel apropiado, según los criterios propuestos más arriba.

La dificultad principal de este sistema radicaría en su costo. El cuadro 5 presenta estimaciones, como proporción del PIB, de un beneficio universal no contributivo para todos los mayores de 65 años, considerando diferentes opciones para el nivel del beneficio y el grado de avance de los países en la transición demográfica. En el cuadro se estiman los costos de tres posibles alternativas para definir el beneficio básico universal: i) una pensión orientada a garantizar a los mayores de 65 años un ingreso equivalente a la línea de indigencia, que sólo alcanza a cubrir los gastos de alimentación, ii) otro equivalente a la línea de pobreza, y iii) un beneficio igual al salario mínimo. Esta última es la opción más onerosa.

Como allí se aprecia, para los países que se encuentran en las etapas iniciales de la transición demográfica el costo de un beneficio universal destinado a evitar la pobreza en la vejez alcanza en promedio el $3 \%$ del PIB, pese a que la población elegible no alcanza aún proporciones muy elevadas; tan significativo costo se debe a que en estos países el producto per cápita está entre los menores de la región. Para los

\footnotetext{
11 Existen regímenes universales sin prueba de medios en Nueva Zelandia, Namibia, Botswana y Mauricio. Otros regímenes exigen dicha prueba (véase Willmore, 2001).
} 
América Latina: Estimaciones del costo de un beneficio universala

\begin{tabular}{cc|ccc|c|c|c}
\hline País & $\begin{array}{c}\text { Población } \\
\text { mayor de } \\
65 \text { años }\end{array}$ & $\begin{array}{c}\text { Línea de } \\
\text { indigencia } \\
\text { urbana }\end{array}$ & $\begin{array}{c}\text { Línea de } \\
\text { pobreza } \\
\text { urbana }\end{array}$ & $\begin{array}{c}\text { Salario } \\
\text { mínimo }\end{array}$ & $\begin{array}{c}\text { PIB per } \\
\text { cápita }\end{array}$ & $\begin{array}{c}\text { Costo, como \% del PIB, de una } \\
\text { pensión universal equivalente a: }\end{array}$ & $\begin{array}{c}\text { Ingresos } \\
\text { corrientes } \\
\text { del gobierno } \\
\text { central }\end{array}$ \\
\hline
\end{tabular}

Países en transición

incipiente o moderada

\begin{tabular}{|c|c|c|c|c|c|c|c|c|}
\hline Nicaragua & 3.1 & 26.3 & 52.7 & & 471.2 & 2.1 & 4.2 & \\
\hline Honduras & 3.4 & 39.3 & 78.6 & 69.1 & 691.2 & 2.3 & 4.6 & 4.1 \\
\hline Bolivia & 4.0 & 28.0 & 56.1 & 55.7 & 959.7 & 1.4 & 2.8 & 2.8 \\
\hline Guatemala & 3.5 & 40.7 & 81.5 & 73.5 & 1547.6 & 1.1 & 2.2 & 2.0 \\
\hline Paraguay & 3.5 & 42.0 & 83.9 & 178.3 & 1602.7 & 1.1 & 2.2 & 4.7 \\
\hline El Salvador & 5.0 & 33.5 & 66.9 & 122.1 & 1736.8 & 1.2 & 2.3 & 4.2 \\
\hline
\end{tabular}

Países en plena

transición

Ecuador

Colombia

R. Dominicana

Venezuela

Panamá

Costa Rica

Brasil

México

$\begin{array}{rrrrrr}4.7 & 19.3 & 38.5 & 52.5 & 1 & 404.4 \\ 4.7 & 37.3 & 74.6 & 139.1 & 2 & 267.8 \\ 4.5 & 42.1 & 84.3 & & 2 & 479.0 \\ 4.4 & 78.8 & 155.9 & 174.7 & 3036.8 \\ 5.5 & 40.7 & 81.4 & 25.5 & 3264.4 \\ 5.1 & 37.5 & 75.1 & 217.4 & 3625.2 \\ 5.2 & 26.7 & 66.2 & 74.5 & 4220.6 \\ 4.7 & 56.8 & 113.6 & 85.6 & 4 & 583.4\end{array}$

$\begin{array}{llll}0.8 & 1.5 & 2.1 & 19.6 \\ 0.9 & 1.9 & 3.5 & 10.8 \\ 0.9 & 1.8 & & 15.3 \\ 1.4 & 2.7 & 3.0 & 16.3 \\ 0.8 & 1.6 & 0.5 & 20.0 \\ 0.6 & 1.3 & 3.7 & 12.5 \\ 0.4 & 1.0 & 1.1 & 11.4 \\ 0.7 & 1.4 & 1.1 & 13.5\end{array}$

Países en transición

avanzada

Chile

Uruguay

Argentina

\begin{tabular}{rrrrrrrrr}
7.2 & 40.9 & 81.8 & 163.1 & 5128.5 & 0.7 & 1.4 & 2.7 & 21.0 \\
12.9 & 56.4 & 112.9 & 88.6 & 5930.0 & 1.5 & 2.9 & 2.3 & 19.2 \\
9.7 & 71.6 & 143.3 & 200 & 7434.9 & 1.1 & 2.2 & 3.1 & 10.8 \\
\hline
\end{tabular}

Fuente: Elaboración de la CEPAL, basada en cifras oficiales.

a Según datos de 1999.

b Excluye las contribuciones a la seguridad social. Descontados los aportes a gobiernos locales.

países en plena transición el costo se reduce, llegando en promedio al $1.7 \%$ del PIB, porque los niveles de ingreso más que compensan la mayor incidencia de población mayor de 65 años en comparación con el primer grupo de países. Por último, el costo se eleva nuevamente para los países en transición demográfica avanzada, donde llega a $2.2 \%$, debido a la mayor población elegible.

La principal fuente de recursos para un programa de esta naturaleza son los ingresos corrientes del gobierno central, los que en promedio representan un $16 \%$ del PIB. ${ }^{12}$ Comparando esta cifra con los costos estima-

\footnotetext{
${ }^{12}$ Las cifras relativas a la recaudación de ingresos corrientes suelen ser difíciles de comparar entre países. Las incluidas en el cuadro 5 no dan cuenta de los ingresos por concepto de utilidades de las empresas públicas. Asimismo, se presentan descontadas las transferencias a los gobiernos locales, las que son más importantes en los
}

dos, vemos que un programa de cobertura universal que ofreciera un beneficio más bien modesto, equivalente al nivel de la línea de pobreza, demandaría aproximadamente entre el $10 \%$ y el $18 \%$ de los ingresos corrientes del gobierno central. ${ }^{13}$ Se deduce, en consecuencia, que para la mayoría de los países de la región un programa de carácter universal como el ana-

países con ordenamiento federativo. Por último, dado que el grado de participación privada en la previsión social difiere de un país a otro, de los datos se han deducido las contribuciones a la seguridad social.

13 Se ha empleado como fuente de recursos el ingreso corriente, descontadas las contribuciones a la seguridad social, por dos razones. En primer lugar, no corresponde emplear los ingresos de capital en el financiamiento de un gasto corriente recurrente (las pensiones). En segundo lugar, este programa no busca sustituir al régimen contributivo, el que continuaría siendo financiado por esas contribuciones. 
lizado podría ejercer una gran presión sobre los recursos públicos, que sería agravada por el grado de avance en la transición demográfica y las legítimas demandas de beneficios superiores al ingreso de pobreza.

La confrontación de los dos hechos claves señalados hasta aquí (la desprotección de quienes por diversas razones no pueden ahorrar para la vejez y la escasez de recursos públicos) obliga en América Latina a que el diseño del sistema de pensiones sea mixto. Por una parte, la escasez de recursos hace indispensable que, mediante un diseño institucional apropiado, se apoye la capacidad de ahorro para la vejez de quienes la tienen y se libere así al Estado de esa responsabilidad, sobre todo respecto de los grupos más acomodados. Por otra parte, el objetivo de evitar la desprotección durante la vejez de quienes enfrentan obstáculos estructurales para participar en los sistemas, junto a la mencionada escasez de recursos públicos, ponen de relieve la importancia de los regímenes no contributivos y la necesidad de que ellos se focalicen en los grupos más pobres.

En lo que toca a los regímenes contributivos, de la baja densidad de las cotizaciones de los grupos de bajo ingreso y de las mujeres se desprende que los regímenes con estrecha vinculación entre aportes y beneficios no podrán ofrecer a estos grupos un buen nivel de reemplazo del ingreso y que aproximadamente un $45 \%$ de los afiliados serán elegibles para recibir una pensión pensión mínima garantizada (Arenas de Mesa, 1999). En otras palabras, en un régimen de pensiones con contribuciones definidas estos grupos por sí solos quedarían subprotegidos y el Estado debería hacer frente a una importante carga por concepto de pensiones garantizadas.

Estas observaciones han llevado a la propuesta, ya incorporada por ciertos países en el diseño de sus sistemas, de incluir el financiamiento solidario de un beneficio básico. Así, las reformas en Argentina y Uruguay, si bien incluyen un componente de capitalización individual, mantienen un beneficio básico financiado mediante el reparto, lo que confiere cierto grado de solidaridad a su financiamiento. ${ }^{14}$ En este caso cabe adoptar, como se señaló más atrás, las precauciones necesarias para evitar comportamientos estratégicos de parte de los participantes que puedan afectar la solvencia del componente de reparto.

\footnotetext{
${ }^{14}$ En el proceso de reformas al sistema de previsión social en Costa Rica también se han formulado propuestas de mantener un componente de financiamiento solidario de un beneficio básico.
}

El sistema descrito es potencialmente superior a un régimen basado sólo en cuentas individuales, por cuanto permite una mayor diversificación de los riesgos. En efecto, los regímenes de capitalización individual concentran los riesgos (financieros, de desempleo y de discontinuidad de ingresos) en el participante individual, permitiendo una diversificación únicamente financiera. Por su parte, los regímenes de reparto están expuestos al riesgo demográfico (alzas en las tasas de dependencia) y tienen pocas posibilidades de diversificación financiera (porque la acumulación de fondos es por lo general baja); pero en ellos puede haber cierta diversificación intergeneracional, por cuanto se comparten los riesgos. Entonces, una combinación de ambos podría proveer una mejor diversificación de riesgos, tanto desde el punto de vista financiero como del intergeneracional. ${ }^{15}$

Por último, los sistemas de pensiones son incompletos si carecen de mecanismos, probablemente separados funcionalmente, para hacer frente a las discontinuidades de aportes originadas en el desempleo y la maternidad. Recientemente ha resurgido en la región el interés por establecer sistemas de seguros de desempleo. La provisión de ingresos durante episodios de desempleo también debiera contemplar, al menos durante cierto período, la continuación de los aportes a los sistemas de pensiones, con cargo a los recursos de ese seguro. De este modo, por la vía de mantener la continuidad de los aportes, se estaría evitando que pérdidas transitorias de ingreso presente se transformen en pérdidas permanentes de ingreso futuro (menores pensiones), y se reduciría la carga fiscal de servir las garantías de pensiones mínimas.

\section{b) Reducir desincentivos a participar en los sistemas} de pensiones contributivos

Una segunda línea de acción hace hincapié en la reducción del costo alternativo de participar en sistemas contributivos de pensiones para los trabajadores independientes y por cuenta propia, y en el derecho de los aportantes a elegir según sus preferencias entre riesgo y rentabilidad.

Conforme a este enfoque, lo que desincentiva la ampliación voluntaria de la cobertura de estos sistemas es su falta de flexibilidad y la escasa diversificación de los productos que ofrecen, factores que impiden a

\footnotetext{
15 Como la experiencia reciente enseña, ambos tipos de instituciones, privadas y públicas, están afectas al riesgo de interferencia política, por lo que ningún diseño posibilita diversificar ese riesgo.
} 
los cotizantes influir en el destino de sus ahorros. Si bajo ciertas condiciones y límites se permitiera acceder a los fondos acumulados para poder destinarlos a usos altamente valorados por sus propietarios, tendería a moderarse el sesgo anticobertura que deriva de la irrevocabilidad o ausencia de liquidez de los aportes, pero probablemente se reduciría la acumulación.

El propósito de restringir el acceso a los fondos es, teóricamente, evitar su desvío hacia usos que podrían poner en peligro la obtención de pensiones adecuadas. No obstante, el desincentivo que significa esa inaccesibilidad puede también traducirse en una acumulación menor. Para paliar lo anterior, así como para cautelar los niveles futuros de pensiones, se podrían establecer límites a los retiros, así como también opciones para su reintegro. Además, para evitar comportamientos de riesgo moral ante la presencia de garantías de pensiones mínimas en el régimen contributivo, el acceso a los fondos debiera significar la pérdida de esa garantía, a menos que esos recursos sean luego reintegrados.

En segundo lugar, los aportantes deberían tener la posibilidad de ejercer sus preferencias entre riesgo y rentabilidad a lo largo de todo el período de afiliación, eligiendo no sólo entre diversas administradoras sino también entre diversos fondos con distintas composiciones de activos, para hacer más atractiva la afiliación a quienes tienen más aversión al riesgo. Desde el punto de vista de los trabajadores dependientes cuya afiliación y cotización es obligatoria, el tener acceso a diferentes productos es una compensación parcial por la obligatoriedad del sistema. En otras palabras, dado que el aporte tiene carácter obligatorio, es al menos equitativo y justo que los cotizantes no estén expuestos a más riesgos que los que ellos juzgan apropiados. Recientemente Chile introdujo reformas en su sistema de pensiones que se orientan en este sentido.

Otro desincentivo a la afiliación de los trabajadores independientes radica en las imperfecciones del sistema tributario. A diferencia de los trabajadores dependientes, en ciertos casos (el de Chile, por ejemplo) los independientes no pueden deducir de la base tributaria sus contribuciones al sistema de pensiones, lo que introduce un sesgo de doble tributación, por cuanto se grava tanto el ahorro como el ingreso que se obtiene más tarde como pensión (Arenas de Mesa, 2000).

c) Reducir la miopía en relación a las necesidades de ahorro para la vejez.

La miopía en las decisiones de consumo y ahorro, como fuente de baja adscripción, ha dado pie al establecimiento de la afiliación obligatoria para trabajadores tanto dependientes como independientes. Desafortunadamente, no existen indicios para afirmar que esta medida contribuye a elevar la cobertura. Habría que considerar la aplicación de medidas orientadas a educar a los potenciales participantes, junto con otras tendientes a hacer de los sistemas de pensiones una opción de ahorro más atrayente. No obstante, ni siquiera en países desarrollados las experiencias con iniciativas de información y educación sobre las necesidades de ahorro para la vejez han arrojado resultados alentadores. ${ }^{16}$

\section{d) Alternativas para elevar la cobertura entre las mujeres}

Como ya se señaló, diversos factores se conjugan para crear una situación de baja cobertura entre las mujeres. El debate en torno a estrategias para superar esta situación, teniendo en cuenta los rasgos estructurales del mercado laboral y de los sectores públicos de la región, es muy incipiente. Aquí sólo abordaremos los temas vinculados al aumento de la cobertura, dado que los relativos a la calidad de los beneficios que perciben las mujeres y a otros aspectos de equidad escapan a los límites de este artículo.

En los países desarrollados, los temas de equidad de género en la seguridad social cobraron mayor relieve en la década de 1990. Aspectos como las diferencias de edad de retiro, el crédito en años cotizados para las mujeres que cuidan a niños y ancianos, la cobertura de las mujeres que trabajan en la casa, en labores de jornada parcial, en el servicio doméstico o en trabajos estacionales como los agrícolas, fueron analizados e incorporados en las reformas a los sistemas de seguridad social en la década de 1990.

De los factores determinantes de la baja cobertura ya examinados, se deduce que los regímenes contributivos y las reformas a ellos serán de poca utilidad para ampliar la cobertura entre las mujeres. La menor participación de ellas en el mercado laboral y su desventajosa inserción laboral, particularmente en los grupos de menores ingresos, tornan ineficaces los regímenes

\footnotetext{
${ }^{16}$ En su edición del 15 de abril del 2002 el diario Financial Times, en su versión electrónica, informó que una experiencia piloto llevada a cabo por el gobierno y los fondos de pensiones del Reino Unido, consistente en informar a miles de contribuyentes respecto de las magras pensiones que recibirían si no incrementaban sus contribuciones, ocasionó una respuesta positiva en menos del 10\% de los casos.
} 
basados en la existencia de una relación laboral formal y estable. Esto implica que quizás los regímenes no contributivos, con beneficios que eviten el riesgo moral y focalizados en quienes no posean capacidad de ahorro ni otros ingresos para su vejez, sean la única alternativa viable para las mujeres de menores ingresos. Dado que las mujeres tienen una mayor representación entre los ausentes del mercado de trabajo y los que tienen empleo informal, este tipo de programa ampliaría implícitamente y en mayor medida la cobertura entre ellas.

Se han formulado diversas propuestas en torno a establecer regímenes especiales para ciertas situaciones en las cuales la presencia de mujeres de bajos ingresos es mayoritaria, como es el caso de algunas actividades con alta estacionalidad o variabilidad. Recientemente,

\section{IV}

\section{Conclusión}

Pese a la insuficiente consistencia y comparabilidad de los datos acerca de la cobertura de los sistemas de pensiones, resulta evidente que ella está fuertemente determinada por factores de estructura económica y que los sistemas contributivos enfrentan serios obstáculos para incluir a importantes segmentos de la población. El nivel del PIB per cápita y la importancia de los segmentos informales y agrícolas del mercado de trabajo aparecen como los principales factores que determinan la cobertura posible de los regímenes contributivos. La experiencia reciente no permite afirmar que la introducción de sistemas privados con un mayor vínculo entre aportes y beneficios sea un instrumento decisivo en la ampliación de la cobertura, aunque sí hace un aporte positivo a la solvencia del régimen. Para enfrentar el desafío de la universalidad de la protección social, evitando la pobreza en la vejez, se requiere un diseño distinto.

Lo expuesto conduce a afirmar que para proteger a toda la población se necesitará una adecuada combinación de regímenes contributivos y no contributivos (es decir, de elementos de seguros sociales y de seguridad social). Dado el costo en recursos públicos que tienen los sistemas no contributivos - estimados en el cuadro 5 en magnitudes significativas del PIB- su ampliación debe ser gradual y estar focalizada en los grupos pobres sin capacidad de ahorro. Asimismo, sus en Chile se propuso que las trabajadoras temporeras pudieran optar a un beneficio mínimo en condiciones que se ajustan más a su realidad laboral. ${ }^{17}$ Este tipo de régimenes especiales es positivo en tanto se focalice en grupos de mujeres de menores ingresos y continúe incentivando su ahorro para la vejez. No obstante, si se restringen sólo a cierto tipo de actividades productivas, tienden a reducir la portabilidad de los beneficios y disminuyen la movilidad laboral, lo cual es un efecto adverso tanto desde el punto de vista del diseño de un sistema de pensiones como desde la óptica de la asignación de recursos productivos. Aún más, la proliferación de regímenes especiales podría reproducir los problemas de fraccionamiento, desigualdad y desequilibrio financiero que en el pasado afectaron a los sistemas de pensiones de la región. beneficios deben ser cuidadosamente establecidos para no crear incentivos inadecuados que redunden en el deterioro de los regímenes contributivos y/o del ahorro para la vejez. Además, los regímenes no contributivos aparecen como la principal opción para expandir la cobertura de las mujeres, cuya desfavorable inserción laboral reduce sus posibilidades de beneficiarse de sistemas basados en relaciones laborales formales.

Por otra parte, una importante proporción de los adscritos a los regímenes contributivos resulta subprotegida, por efectos de la variabilidad en la situación de empleo, y las mujeres en especial enfrentan barreras para lograr un historial contributivo continuo. ${ }^{18} \mathrm{La}$ inclusión en los sistemas de pensiones de un componente de reparto que financie solidariamente un beneficio básico, en conjunto con un régimen de ahorro, es por el momento la principal respuesta a este problema que, según las proyecciones, será muy grave en el futuro.

En suma, para hacer frente al desafío de dar cobertura universal a la población se requiere una combinación de seguros sociales, instituciones de seguridad social y solidaridad en el financiamiento de una

\footnotetext{
${ }^{17}$ Los análisis de factibilidad financiera señalaron que la propuesta resultaba en un régimen oneroso, por lo que aún está en estudio una propuesta definitiva.

${ }^{18}$ Véase, entre otros, Arenas de Mesa (1999) y Uthoff (2001).
} 
parte de los beneficios. Un sistema mixto, que incluya componentes privados y públicos (contributivos y no contributivos), con beneficios financiados sobre la base de ahorro y de fondos solidarios y tributarios, resulta superior a otros que no toman en cuenta la naturaleza de los mercados de trabajo de la región.

\section{Bibliografía}

Acosta, Olga Lucía y Ulpiano Ayala (2002): Políticas para promover una ampliación de la cobertura de los sistemas de pensiones en Colombia, serie Financiamiento del desarrollo, $\mathrm{N}^{\circ} 118$, LC/L.1724-P, Santiago de Chile, Comisión Económica para América Latina y el Caribe (CEPAL), abril. Publicación de las Naciones Unidas, $\mathrm{N}^{\circ}$ de venta: S.02.II.G.39.

(2001): Reformas pensionales y costos fiscales en Colombia, serie Financiamiento del desarrollo, N 116, LC/L. 1630P, Santiago de Chile, Comisión Económica para América Latina y el Caribe (CEPAL), noviembre, Publicación de las Naciones Unidas, $\mathrm{N}^{\circ}$ de venta: S.01.II.G.167.

Acuña, Rodrigo y Augusto Iglesias (1991): Chile: experiencia con un régimen de capitalización, 1981-1991, Santiago de Chile, Comisión Económica para América Latina y el Caribe (CEPAL)

Arenas de Mesa, Alberto (2000): Cobertura previsional en Chile: Lecciones y desafíos del sistema de pensiones administrado por el sector privado, serie Financiamiento del desarrollo, $\mathrm{N}^{\circ} 105$, LC.L.1457-P, Santiago de Chile, Comisión Económica para América Latina y el Caribe (CEPAL), diciembre. Publicación de las Naciones Unidas, $\mathrm{N}^{\circ}$ de venta: S.00.II.G.137.

(1999): Efectos fiscales del sistema de pensiones en Chile: proyecciones del déficit previsional 1999-2037, inédito.

Arenas de Mesa, Alberto y Héctor Hernández (2001): Análisis, evolución y propuestas de ampliación de la cobertura del sistema civil de pensiones en Chile, Cobertura previsional en Argentina, Brasil y Chile, Fabio M. Bertranou (comp.), Santiago de Chile, Oficina Internacional del Trabajo (OIT).

Barr, Nicholas (2000): Reforming Pensions: Myths, Truths and Policy Choices, IMF Working Paper, $\mathrm{N}^{\circ} 139$, Washington, D.C., Fondo Monetario Internacional (FMI), agosto.

CEPAL (Comisión Económica para América Latina y el Caribe) (2002): América Latina y el Caribe: estimaciones y proyecciones de población 1950-2050, Boletín demográfico, año 35, $\mathrm{N}^{\circ}$ 69, LC/G.2152-P, División de Población de la CEPAL-Centro Latinoamericano y Caribeño de Demografía (CELADE), enero. Publicación de las Naciones Unidas, $\mathrm{N}^{\circ}$ de venta: S.01.II.G.179.

(2001): Panorama social de América Latina 2000-2001, LC.G.2138-P, Santiago de Chile. Publicación de las Naciones Unidas, $\mathrm{N}^{\circ}$ de venta: S.01.II.G.141.

(2000): Panorama social de América Latina 1999-2000, LC/G.2068-P, Santiago de Chile. Publicación de las Naciones Unidas, $\mathrm{N}^{\circ}$ de venta: S.00.II.G.18.

Diamond, Peter (2001): Towards an Optimal Social Security Design, CeRP Working Paper, Centro de Investigación sobre Políticas de Pensiones y Bienestar (CeRP), abril.

Fox, Louise y Edward Palmer (2001): Pension Reform in Europe in the 90's and Lessons for Latin America, serie Financiamiento del desarrollo, $\mathrm{N}^{\circ} 114$, LC/L.1628-P, Santiago de Chile, Comisión Económica para América Latina y el Caribe (CEPAL), noviembre. Publicación de las Naciones Unidas, $\mathrm{N}^{\circ}$ de venta: E.01.II.G.166.

Hernández Licona, Gonzalo (2001): Políticas para promover una ampliación de la cobertura de los sistemas de pensiones: el caso de México, serie Financiamiento del desarrollo, $\mathrm{N}^{\circ} 107$ LC/L.1482-P. Santiago de Chile, Comisión Económica para América Latina y el Caribe (CEPAL), enero. Publicación de las Naciones Unidas, $\mathrm{N}^{\circ}$ de venta. S.01.II.G-15.

Jiménez, Luis Felipe y Nora Ruedi (1998): Determinantes de la desigualdad entre los lugares urbanos, Revista de la CEPAL, $\mathrm{N}^{\circ}$ 66, LC/G. 2049-P, Santiago de Chile, Comisión Económica para América Latina y el Caribe (CEPAL), diciembre.
Lora, Eduardo y Carmen Pagés (2000): Hacia un envejecimiento responsable: Las reformas de los Sistemas Pensionales en América Latina, Washington, D.C., Banco Interamericano de Desarrollo (BID), octubre.

Mastrángelo, Jorge (1999): Políticas para la reducción de costos en los sistemas de pensiones: el caso de Chile, serie Financiamiento del desarrollo, $\mathrm{N}^{\circ}$ 86, LC/L. 1246-P, Santiago de Chile, Comisión Económica para América Latina y el Caribe (CEPAL), septiembre. Publicación de las Naciones Unidas, $\mathrm{N}^{\circ}$ de venta: S.99.II.G.36.

Mesa-Lago, Carmelo y Alberto Arenas de Mesa (1998): The Chilean pension system: evaluation, lessons and challenges, Do Option Exist?: The Reform of Pension and Health Systems in Latin America, María Amparo Cruz Saco y Carmelo Mesa-Lago (comps.), Pittsburgh, Pennsylvania, University of Pittsburgh Press.

Mesa-Lago, Carmelo; María Amparo Cruz Saco y Lorena Zamalloa (1990): Determinantes de los costos y la cobertura del seguro-seguridad social en la América Latina, El trimestre económico, vol. $57, \mathrm{~N}^{\circ} 1$.

OIT (Organización Internacional del Trabajo) (2000): Panorama laboral de América Latina y el Caribe, 2000, Lima, Oficina Regional de la OIT para América Latina y el Caribe.

Packard, Truman y Naoko Shinkai (s/f): The reach of social security in Latin America: a look at the determinants of coverage in the region, Washington, D.C., Banco Mundial/Banco Interamericano de Desarrollo (BID).

Palacios, Robert y Montserrat Pallares-Miralles (2000): International Patterns of Pension Provision, Washington, D.C., Banco Mundial, abril.

Rabelo, Flavio (2001): Pension Systems for Public Employees in Brazil: Options for Reform, inédito.

Rodríguez, Adolfo y Fabio Durán (2000): Costos e incentivos en la organización de un sistema de pensiones, serie Financiamiento del desarrollo, $N^{\circ}$ 98, LC./L.1388-P, Santiago de Chile, Comisión Económica para América Latina y el Caribe (CEPAL), junio. Publicación de las Naciones Unidas, $\mathrm{N}^{\circ}$ de venta: S.00.II.G.63.

Schmidt-Hebbel, Klaus (1998): Does Pension Reform Really Spur Productivity and Growth?, Documento de Trabajo, № 33, Santiago de Chile, Banco Central de Chile.

Schulthess, Walter y Gustavo Demarco (1993): Sistema de pensiones en América Latina. Argentina: Evolución del sistema nacional de previsión social y propuesta de reforma, Colección Estudios Políticos y Sociales, Santiago de Chile, S.R.V. Impresos.

Superintendencia de Administradoras de Fondos de Pensiones (2002): Boletín estadístico, Santiago de Chile, enero-abril.

Uthoff, Andras (2001): La reforma del sistema de pensiones en Chile y desafíos pendientes, serie Financiamiento del desarrollo, № 112, LC/L.1575-P, Santiago de Chile, Comisión Económica para América Latina y el Caribe (CEPAL), junio. Publicación de las Naciones Unidas, $\mathrm{N}^{\circ}$ de venta: S.01.II.G.118.

Uthoff, Andras y Raquel Szalachman (1991, 1992, 1994): Sistema de pensiones en América Latina y el Caribe, 3 volúmenes, Santiago de Chile, Comisión Económica para América Latina y el Caribe (CEPAL).

Willmore, Larry (2001): Universal Pensions in Low-Income Countries, Nueva York, División de Economía y Administración del Sector Público, Departamento de Asuntos Económicos y Sociales (DESA), Naciones Unidas. 